\title{
Brain systems for encoding and retrieval of auditory-verbal memory An in vivo study in humans
}

P. C. Fletcher, ${ }^{1,3}$ C. D. Frith, ${ }^{1,2}$ P. M. Grasby, ${ }^{1,3,4}$ T. Shallice, ${ }^{2}$ R. S. J. Frackowiak ${ }^{1,4}$ and R. J. Dolan ${ }^{1,3,4}$

'MRC Cyclotron Unit, Hammersmith Hospital, the ${ }^{2}$ Psychology Department, University College of London, the ${ }^{3}$ Royal Free Hospital School of Medicine and the ${ }^{4}$ National Hospital for Neurology and Neurosurgery, London, UK
Correspondence to: Paul Fletcher, MRC Cyclotron Unit, Hammersmith Hospital, Ducane Road, London W12 OHS, $U K$

\begin{abstract}
Summary
Long-term auditory-verbal memory comprises, at a neuropsychological level, a number of distinct cognitive processes. In the present study we determined the brain systems engaged during encoding (experiment 1) and retrieval (experiment 2) of episodic auditory-verbal material. In the separate experiments, PET measurements of regional cerebral blood flow ( $r C B F)$, an index of neural activity, were performed in normal volunteers during either the encoding or the retrieval of paired word associates. In experiment 1 , a dual task interference paradigm was used to isolate areas involved in episodic encoding from those which would be concurrently activated by other cognitive processes associated with the presentation of paired associates, notably priming. In experiment 2, we used the cued retrieval of paired associates from episodic or from semantic memory in order to isolate
\end{abstract}

the neural correlates of episodic memories. Encoding of episodic memory was associated with activation of the left prefrontal cortex and the retrosplenial area of the cingulate cortex, while retrieval from episodic memory was associated with activation of the precuneus bilaterally and of the right prefrontal cortex. These results are compatible with the patterns of activation reported in a previous PET memory experiment in which encoding and retrieval were studied concurrently. They also indicate that separate brain systems are engaged during the encoding and retrieval phases of episodic auditory-verbal memory. Retrieval from episodic memory engages a different, but overlapping, system to that engaged by retrieval from semantic memory, a finding that lends functional anatomical support to this neuropsychological distinction.

Keywords: PET; episodic memory; encoding; retrieval

\section{Introduction}

Neuropsychological, neuroanatomical and neurophysiological studies indicate that human auditory-verbal memory is a multicomponent process. From a neuropsychological perspective, memory processes are classically divided into short- and longterm components, referring, respectively, to limited capacity stores of brief duration and a system of theoretically unlimited capacity enduring over the lifetime of an individual (Baddeley, 1990). Long-term memory can, itself, be sub-divided into components accessible to consciousness (declarative or explicit memory) and components which are inaccessible to consciousness (procedural or implicit memory). Within the former, a division into semantic and episodic memory systems has been proposed (Furlong, 1951; Tulving, 1972, 1983). Semantic memory has been described as 'a mental thesaurus' involved in the comprehension, organization and concatenation of verbal symbols and in non-verbal understanding and knowledge of our environment. Episodic memory deals with the occurrence of episodes or events which have an autobiographical context (Tulving, 1972, 1983). Psychological evidence has provided tentative support for the distinction (Tulving, 1983; Baddeley, 1990). Nevertheless, the neuropsychological distinction between episodic and semantic memory, though widely held, has not gained universal acceptance (Cermak and Craik, 1979; Nickerson, 1980). 
From a neuroanatomical perspective, a number of separate brain areas have been implicated in long-term memory function (Amaral, 1987). These include medial temporal lobe structures (Milner, 1966; Weizkrantz, 1982), the diencephalon (Parkin, 1984), the prefrontal cortex (Goldman-Rakic, 1987) and the retrosplenial area of the cingulate gyrus (Valenstein et al., 1987; Rudge and Warrington, 1991). A neuroanatomical basis for a distinction between episodic and semantic memory has not, however, been fully described. Baddeley has concluded that it has never been clear that the semanticepisodic definition represented separate systems rather than two modes of operation of the same system' (Baddeley, 1990).

The pattern of deficits seen in the amnesic syndrome following damage to the medial temporal lobes or the diencephalon results in a deficit of episodic memory while semantic memory remains relatively intact (Parkin, 1984). A more general loss of semantic memory with preservation of episodic memory has also been reported in a woman with predominantly left mesial temporal lobe damage following encephalitis (De Renzi et al., 1987). Such findings have been interpreted as providing anatomical evidence for a dissociation between brain systems involved in semantic and episodic memory (McCarthy and Warrington, 1990). However, it seems likely that there is a gradual acquisition process from episodic to semantic memory since a semantic memory must, at some stage, have been encoded as an episodic memory. A degree of overlap between these two descriptively different types of memory is likely and the concept of a strict dichotomy is clearly a conceptual simplification.

A critical distinction within memory function is that between the processes of encoding and retrieval. No satisfactory neuroanatomical basis has been described for these memory processes. Lesions of the monkey hippocampus impair recently acquired, but not more remotely acquired, memory. This has led to the proposal that the hippocampus plays a role in acquisition processes but not in the retrieval of consolidated memory traces (Zola-Morgan and Squire, 1990).

Functional imaging studies of memory systems, particularly using PET, provide an in vivo mode of analysis of hitherto unexplored functional anatomical aspects of memory processing in the intact brain. Although it is true that PET has been used widely in the study of memory, few experiments have looked specifically at episodic long-term memory. Grasby et al. (1993a) sought to isolate activations associated with episodic long-term memory by contrast with short-term memory. Subjects underwent PET scans while performing sub- and supra-span wordlist memory tasks and a widespread neural system involved in long-term memory comprising the pre-frontal cortex (bilaterally), the posterior cingulate cortex and the precuneus was identified. A comparison of the suband supra-span tasks with a rest condition showed common activation of the left anterior cingulate gyrus, the thalamus and the cerebellum. In a separate investigation, using a graded task paradigm, the same authors also showed activation of the hippocampus associated with a measure of memory performance (Grasby et al., 1993b). A further PET study, in which subjects were required to hold up to 10 items in memory, has shown activation of the prefrontal cortex, anterior cingulate and posterior parietal cortex (Petrides et al., 1993). Using previously presented word stems and performing PET scans during the retrieval stage only, increases of regional cerebral blood flow (rCBF) in the right hippocampus, parahippocampal gyrus and the right prefrontal cortex have been observed in a study involving cued recall (Squire et al., 1992).

In general, in the majority of previous PET studies investigators have sought to identify the functional anatomy of memory as a whole, i.e. its encoding and retrieval. A minority of investigators have studied encoding and retrieval separately (Squire et al., 1992; Kapur et al., 1994; Tulving et al., 1994). In the present study, we also sought to examine these processes separately in addition to determining whether episodic and semantic memory systems were anatomically dissociable. Two discrete sets of experiments were therefore carried out. In both, we used the single presentation and retrieval of paired associates as the paradigm for episodic memory. The use of this design enables us to examine encoding and retrieval processes operating fairly continuously throughout the PET scanning period since performance can be close to $100 \%$. However, this makes it necessary to contrast the episodic memory component of the task with that of other memory processes that could potentially make a contribution to performance of the tasks. In work on amnesia, it has been shown that priming can make a major contribution if the paired associate relation is very close (Warrington and Weizkrantz, 1982). While the contribution of priming was reduced by the use of low frequency categoryexemplar pairs, it is necessary to include a control condition where the actual contribution of priming in the particular paradigm used can be allowed for. In the first experiment, involving encoding, a dual task approach was used in order to isolate episodic memory encoding from concurrent priming processes. It is known that a structurally unrelated, but attentionally demanding task interferes with encoding of long-term memory but not with priming (Baddeley et al., 1984; Jacoby et al., 1993). The use of a difficult concurrent task should therefore selectively attenuate the activation of the neural systems engaged during episodic memory encoding but leave any priming processes unaffected. By contrast, episodic memory should be unimpaired when subjects performed a structurally similar, but easy, distraction task. We predicted that incorporating this experimental evidence into a cognitive subtraction paradigm would allow us to isolate brain activity in episodic memory encoding. The cognitive subtraction to be used is represented algebraically in Fig. 1 . In a second experiment, involving retrieval, cued paired associates were used to examine retrieval from episodic memory. It has been shown that a priming task is not associated with activation of any additional brain areas to those seen in a semantic memory task (Squire et al., 1992). 


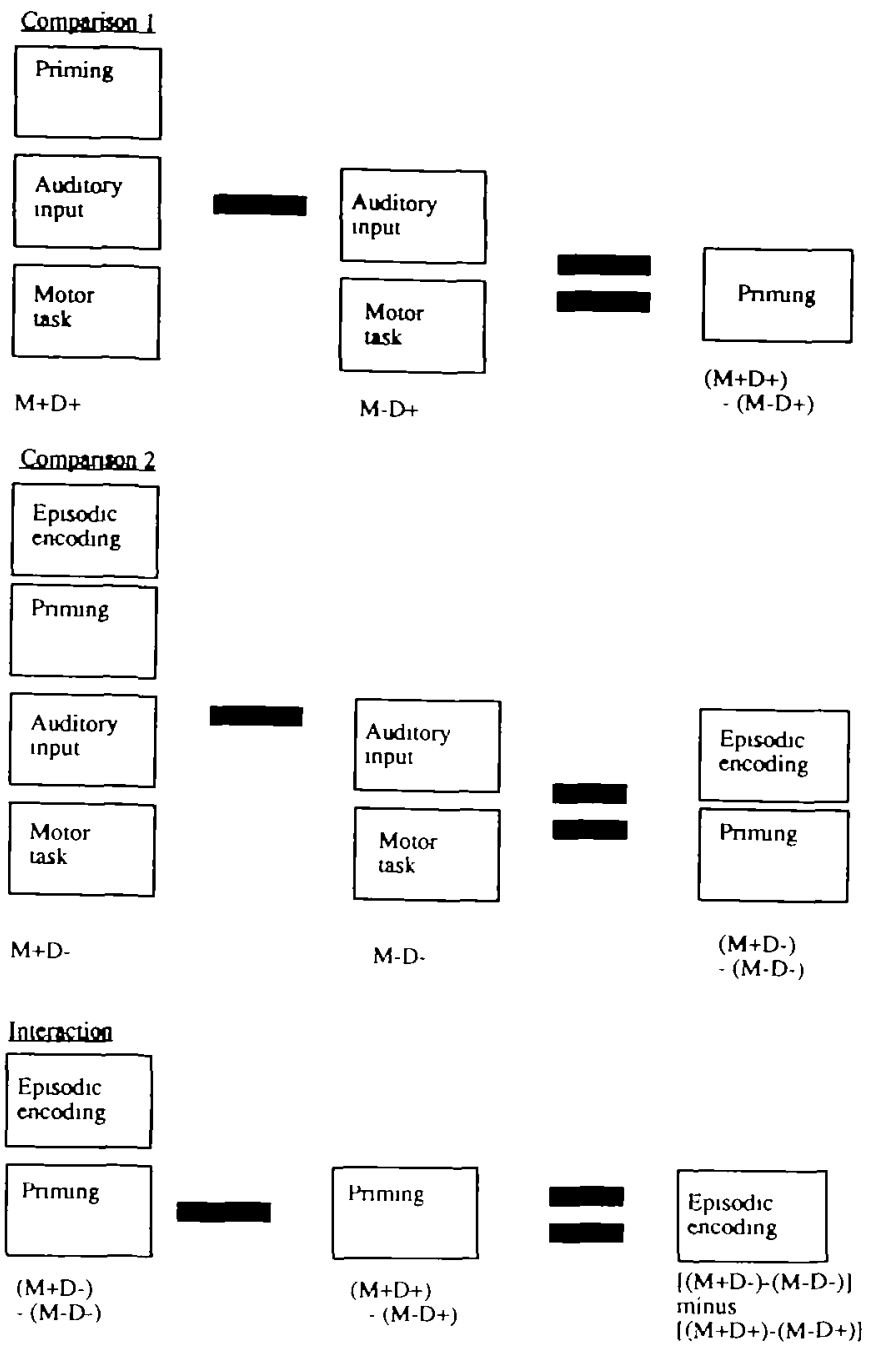

Fig. 1 Diagrammatic representation of comparisons made during expenment 1.

Therefore, in order to identify the confounding semantic memory and priming components of this task we also administered a separate task involving semantic but not episodic memory.

Thus, the aim of this study was primarily to examine whether there are distinct neural systems for encoding into and retrieval from episodic memory. In view of the different processes which predominate at these stages (e.g. organizational strategies at encoding; monitoring/verification strategies at retrieval) we predicted that the pattern of activity seen would differ across experiments 1 and 2 but that, overall, the brain regions seen to be active would be in broad agreement with previous PET experiments on auditory-verbal episodic long-term memory (Squire et al., 1992; Grasby et $a l ., 1993 a, b$ ). We also hypothesized that the episodic and semantic memory tasks would engage different brain regions providing functional anatomical evidence for the validity of the episodic-semantic distinction. A preliminary account of these experiments has already been reported (Shallice et al., 1994).

\section{Material and methods \\ Subjects}

Twelve right-handed male volunteers (age range 21-38 years) took part in the study. All subjects were fit, healthy and free of any significant previous or current medical or neurological illness. The study involved the administration of $7.2 \mathrm{mSv}$ effective dose equivalent of radioactivity per subject and was approved by the Administration of Radioactive Substances Advisory Committee of the Department of Health of the UK. Subjects gave informed, written consent and the study was approved by the joint research ethics committee of the Royal Postgraduate Medical School, Hammersmith Hospital, London.

\section{PET scanning}

Scans of rCBF were obtained using a CTI model 953B-PET scanner (CTI Inc, Knoxville, Tenn., USA) with collimating septa retracted. For each scan, subjects received a $20 \mathrm{~s}$ intravenous bolus of $\mathrm{H}_{215} \mathrm{O}$ at a concentration of $55 \mathrm{mBq} / \mathrm{ml}$ and a flow rate of $10 \mathrm{ml} / \mathrm{min}$ through a forearm cannula. Twelve consecutive PET scans were collected at $10 \mathrm{~min}$ intervals each over a period of $2 \mathrm{~min}$, beginning with a 30 -s background scan before the delivery of the bolus. The integrated radioactivity counts accumulated over the 90-s acquisition period, corrected for background, were used as an index of rCBF. Subjects were scanned in a quiet, darkened room. In the first experiment the subjects' eyes were fixed on a computer screen during scanning; in the second experiment, subjects were studied with their eyes closed.

\section{Memory tasks}

Experiment 1: encoding of episodic memory

Memory task. Prior to scanning, subjects were instructed that, during the scan, they would be read a list of categories and category exemplars and that they should try to remember the exemplars for later recall. Rare category exemplars were chosen (Battig and Montague, 1969) During scanning, subjects were presented a list of 15 categories each paired with a relevant exemplar (e.g. Poet . . . Browning). The pairs were read out by the investigator, who stood beside the scanning couch, at a rate of one per 3 s over a $45-s$ period. In the 5-min period following the presentation/scanning stage, a 'stress and arousal' questionnaire was completed verbally by each subject and then memory performance was assessed. (The questionnaire primarily served the role of preventing list rehearsal.) Categories were then presented at a rate of one per $3 \mathrm{~s}$ and subjects were asked to provide the relevant exemplar at each prompt. If unable to recall an item the subject was instructed to say 'pass'. Subjects' performance, 
in terms of percentage correctly recalled, was recorded. The memory task was repeated six times (each time with a different list of paired associates. An example of one of the lists is given in Appendix 1), three times with an easy and three times with a difficult distracting task (see below). In addition, a control task was administered an equal number of times with both types of distracting tasks. In this, during scanning, the subjects were presented with an identically paced auditory input comprising the words 'one thousand ... two thousand' spoken repeatedly for $45 \mathrm{~s}$ at a rate of one presentation per $3 \mathrm{~s}$.

Distracting task. While performing the memory and control tasks, subjects had received prior instruction in the use of a joy stick placed beside their right hands. They were required, during scanning, to move a cursor, on a directly facing screen, into rectangular boxes appearing randomly in one of four positions around the screen. An interval of 0.25 $s$ accurred between successive appearances of boxes. In order to control for the number of boxes appearing to the subject during each scan, the total time for each trial was kept constant such that, independent of reaction time, the box remained on the screen for a constant period (1.1 s). The number of boxes presented, mean reaction time, standard deviation and number of errors were recorded. An easier distraction task, identical in every other respect, involved the boxes appearing in a predictable way, i.e. appearing clockwise around the four positions on the screen (Fig. 2). It had previously been shown in six subjects studied outside the current experiment, that the more difficult (unpredictable) distracting task impaired memory performance on a category. cued recall paradigm when compared with a category-cued recall in the presence of the easier (predictable) distraction task.

Thus, in experiment 1 , there were four possible combinations of task administered to the subjects. These were, first, the memory task with the difficult distracting task $(\mathrm{M}+\mathrm{D}+)$; secondly, the memory task with the easy distracting task (M+D-); thirdly, the control task with the difficult distracting task (M-D+) and, finally, the control task with the easy distracting task (M-D-). Each combination was administered three times over the 12 scans in a counterbalanced order to control for habituation and order effects.

Statistical comparisons. Taking rCBF distributions of subjects in the four possible conditions, we examined memory-induced cerebral activations in the presence of both the easy and the difficult distracting task. The comparison of rCBF profile in the $M+D+$ task with that in the $M-D+$ task represents the neural activity associated with encoding in the presence of the difficult distractor wherein, as we have described, we predicted attenuation of episodic memory encoding with preservation of normal priming processes. A comparison of the $\mathrm{rCBF}$ distributions in the $\mathrm{M}+\mathrm{D}$ - task with that in the M-D- task represents the neural activity in the
1

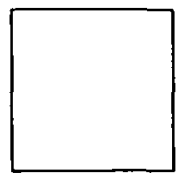

4

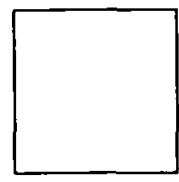

3

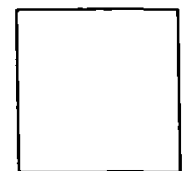

Fig. 2 Manual distracting tasks. On a BBC microcomputer screen placed on a cradle in the centre of the subject's field of vision boxes appeared in one of four positions $(1,2,3$ or 4$)$. The subject was instructed to use a joy-stick, fixed in a convenient position beside his right hand, to move the cursor into the box as rapidly as possible. Each trial lasted a fixed time so that the same number of boxes were seen in all conditions and there was an inter-trial interval of $0.25 \mathrm{~s}$. In the difficult distracting task, the box appeared randomly in positions $1-4$. In the easy distracting task it appeared in successive positions in a predictable order.

presence of an easy distractor, which we predicted would reflect activity associated with priming plus unattenuated episodic encoding. This is represented diagrammatically in Fig. 2. A comparison of the $\mathrm{rCBF}$ profile produced by comparison 1 (i.e. $M+D+$ versus $M-D+$ ) with that produced by comparison 2 (i.e. $M+D$ - versus $M-D-$ ) identifies areas that are specific to the encoding of episodic memory. This comparison may be represented thus: $(M+D$ - versus $M-$ $D-)$ versus $(M+D+$ versus $M-D+)$. We also reversed these comparisons so that we were able to examine those areas showing relative decreases in blood flow associated with performance of the memory encoding tasks.

\section{Experiment 2: retrieval of memory}

In the second experiment, two components of memory retrieval were examined, namely retrieval from semantic and from episodic memory. In the first task, engaging episodic memory, 5 min prior to scanning, subjects were presented with a list of 15 categories, at a rate of one per $3 \mathrm{~s}$ over a 45-s period, each paired with a relevant exemplar. In the 5 min period from the list presentation to the beginning of the scan, a 'stress and arousal' questionnaire was completed verbally by subjects, thus preventing rehearsal in working memory. During scanning, the subjects were prompted with a category, at a rate of one per $3 \mathrm{~s}$ and asked to provide the 
relevant exemplar at each prompt. If unable to recall an item, subjects were instructed to say 'pass'. The second task consisted of retrieval from semantic memory as there was no prior episode of rehearsal. Instead, during scanning, subjects were presented with a list of 15 categories and a prior instruction to provide a relevant exemplar of their own choice for each cued category. Categories were presented at a rate of one per $3 \mathrm{~s}$. If unable to provide an example, subjects were instructed to say 'pass'. The control condition involved verbal repetition, so that subjects were presented with words or phrases of similar characteristics to the categories presented during each of the memory retrieval tasks and instructed simply to repeat these without needing to remember them or to generate any other response (e.g. cue: 'US president' . . . response: 'US president'). The rate of presentation was one item per $3 \mathrm{~s}$. The episodic (E), semantic ( $S$ ) and repetition (R) tasks were presented in four blocks of three as follows: R, E, S, S, E, R, etc. to prevent order and habituation effects. Performance on the two memory retrieval tasks was assessed as the number and percentage of words correctly recalled during each scan.

Statistical comparisons. Three main comparisons of the distribution of rCBF were performed: (i) episodic memory versus word repetition; (ii) semantic memory versus word repetition; and (iii) episodic versus semantic memory. Thus the first comparison represents the neural activity associated with the performance of the combined episodic memory retrieval task. The second comparison represents neural activity associated with retrieval from semantic long-term memory. The final comparison, of episodic versus semantic memory, represents neural activity unique to retrieving episodic memories. Again we performed a comparison to show those areas where memory retrieval was associated with a relative decrease in blood flow.

In this way, we were able to isolate the functional anatomy of encoding and retrieving episodic memories. The tasks are complex because of the difficulty posed by isolating the targeted components of episodic memory behaviourally without contamination by other associated processes.

\section{Data analysis}

Image analysis was performed using statistical parametric mapping (Frackowiak and Friston, 1994) (SPM software, MRC Cyclotron, London, UK) on a SPARC 1 workstation (Sun Microsystems Inc., London, UK) using an interactive image analysis software package (ANALYZE, Biodynamic Research Unit, Mayo Clinic, Rochester, Minn., USA) (Robb and Hanson, 1991). Calculations and image matrix manipulations were performed in PRO MATLAB (Mathworks Inc., New York, USA).

Each $\mathrm{rCBF}$ scan was reconstructed with $3 \mathrm{D}$ algorithms into 31 primary transverse planes which were interpolated to 43 planes to render the voxels approximately cubic $(2.0 \times 2.0 \times 2.0 \mathrm{~mm})$. Subsequently, the total set of scans was realigned as follows: an image was chosen as a reference from the 12 scans of each subject and was then corrected for roll and yaw. The remaining 11 scans were aligned to the reference image using reregistration software (Woods et al., 1992). A mean rCBF image was created for each subject and edited to remove counts representing blood flow to the skull or scalp. The edited mean image was then used as a template to edit the 12 individual realigned images for each subject.

The data were then transformed into a standard stereotactic space (Talairach and Tournoux, 1988; Friston et al., 1989). Such transformation of the data allows for pixel by pixel averaging of data across subjects. A Gaussian filter full width at half maximum $(20 \mathrm{~mm})$ was applied at this stage to smooth each image to accommodate inter-subject differences in gyral and functional anatomy and to suppress high frequency noise in the images thus optimizing the signal to noise ratio. A further Gaussian filter ( $8 \mathrm{~mm}$ full width at half maximum) was applied at the stage of the computation of the statistical parametric maps (SPM) to increase the signal to noise ratio in the data set. The resolution of the resulting images was $\sim 14 \mathrm{~mm}$. Differences in global activity within and between subjects were removed by analysis of covariance (ANCOVA) (Wildt and Ahtola, 1978) on a pixel by pixel basis with global counts as covariate and regional activity across subjects for each task as treatment. This was undertaken as inter- and intra-subject differences in global activity may obscure regional alterations in activity following psychological activations.

For each pixel in stereotactic space the ANCOVA generated a condition specific adjusted mean $\mathrm{rCBF}$ value (normalized to $50 \mathrm{ml} / 100 \mathrm{ml} / \mathrm{min}$ ) and an associated adjusted error variance (Friston et al., 1990). This allowed the planned comparisons of the mean blood flow distributions across all sets of conditions using the $t$ statistic. The resulting sets of $t$ values constituted SMP (Friston et al., 1991).

\section{Results \\ Task performance}

Experiment 1: acquisition of episodic memory

The mean number of words recalled following paired associate presentation during the easy distracting task was $12.5(\mathrm{SD}=1.5)(83.3 \%)$. The performance means across the six subjects varied from 10.33 to 14.33 . This level of performance did not differ significantly from that seen in experiment 2 (when no concurrent motor task was being performed). The mean number of words recalled when the pairs were presented in association with the difficult distracting task was $10.3(\mathrm{SD}=1.3)(68.7 \%)$ with the mean performances across the subjects varying from 8.67 to 12 . The difference in performance associated with the difficult distractor was significantly inferior to that associated with the easy distractor $(P<0.001)$.

[Prior to starting this PET study, we tested the effects of the manual distracting task on the memory performance in a 
group of six healthy volunteers: performance was similar to that found during PET scanning with an average performance during the difficult distracting task of $10.4(\mathrm{SD}=1.6)$ and $13.3(\mathrm{SD}=1.4)$ during the easy distracting task. This was also significant at $P<0.001$. This indicates that subjects were performing normally while in the PET camera.]

\section{Experiment 2: retrieval of episodic memory}

The average number of words recalled during retrieval from episodic memory was $12.1(\mathrm{SD}=2)(80.8 \%)$ with the mean performances across the subjects varying between 10 and 14.5 . This is virtually identical to performance in the same condition when tested in experiment 1 . During the semantic memory task performance was, as expected, $100 \%$ for all subjects.

\section{Experiment 2: distracting tasks}

Performance of the difficult distractor task, during concurrent memory encoding, showed a reaction time of $512.4 \mathrm{~ms}$ $(S D=71)$. During this task, the average number of boxes seen was $38.9(\mathrm{SD}=3.5)$. Performance of the difficult distractor task in the absence of memory encoding showed a reaction time of $452.7 \mathrm{~ms}(\mathrm{SD}=58.7)$. During this task, the average number of boxes seen was $38.6(\mathrm{SD}=2.7)$.

Performance of the easy distractor task in the presence of memory encoding showed a reaction time of $426.2 \mathrm{~ms}$ ( $\mathrm{SD}=$ 83.9). During this task, the average number of boxes seen was $38.1(\mathrm{SD}=3.2)$. Performance of the easy distractor task in the absence of memory encoding showed a reaction time of $366 \mathrm{~ms}(\mathrm{SD}=63)$. During this task, the average number of boxes seen was 39.1 ( $\mathrm{SD}=3.2)$. The difficult distractor task had a significantly slowing effect on the reaction times $[F(1,5)=32.3, P<0.01]$. Memory encoding showed an effect on reaction times which did not reach significance $[F(1,5)=6.0, P=0.058]$.

\section{PET results}

\section{Experiment 1: encoding $r C B F$ increases}

Acquisition of memory in the presence of the difficult distracting task. A comparison of scans in which the subject performed the control (passive listening) task in the presence of the difficult distractor with those of the memory task in the presence of the same, difficult distracting condition, i.e. $(M+D+)$ versus $(M-D+)$ revealed significant activations in two areas: (i) the superior temporal gyri (bilaterally); and (ii) the left anterior cingulate cortex (extending into the left medial frontal gyrus: Brodmann area 9/10). These results are shown, as SPMs, in Fig. 3A.

Acquisition of memory in the presence of a nondistracting task. A comparison of the control task with the memory task, performed in the presence of the easy distractor, i.e. $(M+D-)$ versus ( $M-D-)$, showed similar activations to those seen in the presence of the difficult distractor with, in addition, the retrosplenial area of the posterior cingulate cortex and the left prefrontal contex. These results are shown as SPMs in Fig. 3B.

Activations unique to performance of the memory task in the presence of the easy distractor task. In this comparison, we contrasted patterns of memory-induced activations in the presence and the absence of the distracting task. This comparison can be represented algebraically as follows: $[(M+D-)-(M-D-)]$ versus $[(M+D+)-(M-D+)]$. To constrain the range of pixels analysed, a mask of areas engaged by the performance of the memory task in the presence of the non-distracting task (M+D- versus $M-D-)$ was implemented, i.e. having made this comparison $(M+D-$ versus $M-D-$ ) we stored it as a mask so that when we performed the above comparison looking at the memoryinduced activations with both the distracting tasks, it was constrained to those areas which the data had shown to be involved in the encoding task as a whole. This analysis therefore identifies regions whose activation during encoding are attenuated or augmented by the simultaneous performance of the difficult distractor task. This masked comparison is shown as an SPM in Fig. 4A. Significant activations associated with episodic memory encoding (see Fig. 2) were seen in the left prefrontal cortex and the retrosplenial area of the cingulate cortex. The reverse of this comparison showing those areas activated by the encoding task in the presence of the difficult distractor versus those activated in the presence of the easy distractor, i.e. $[(M+D+)-(M-D+)]$ versus $[(M+D-)-(M-D-)]$, was associated with an increase in activation highlighting the left anterior cingulate cortex alone. This comparison is presented as an SPM in Fig. 4B.

\section{rCBF decreases}

Comparisons of the memory task with the control task, performed in the presence of the easy distractor (i.e. M-Dversus $M+D-$ ) and the difficult distractor (i.e. $M-D+$ versus $M+D+$ ), were also performed to indicate those areas where there is a decrease in blood flow during the encoding tasks. In both cases there was a deactivation of the right prefrontal region, the precuneus, the premotor areas and bilateral parietal regions. The findings for all comparisons, with relevant coordinates from the stereotactic atlas of Talairach and Toumoux (1988), are summarized in Table 1A (rCBF increases), Table 1B (effects of distractor task on memory induced activations) and Table 2 (rCBF decreases).

A potential problem lay in the possibility of the distractor task causing a ceiling effect (and thus disguising any effects associated with memory encoding during the $M+D+$ task). A further comparison was made between the $\mathrm{M}-\mathrm{D}+$ and the $\mathrm{M}-\mathrm{D}-$ conditions to address this. This showed a small area of significant activity in the precuneus (medial parietal area 
A
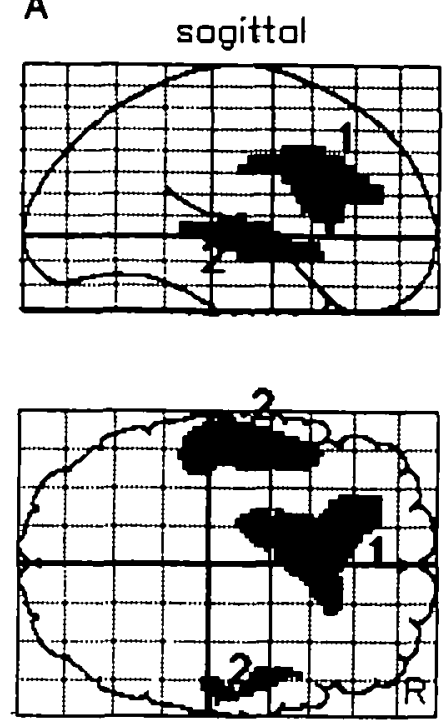

transverse
B
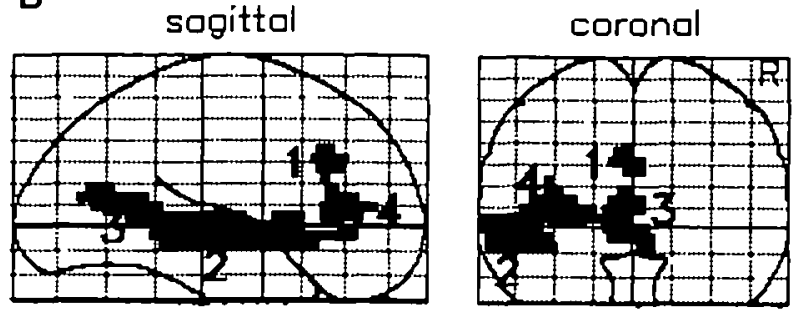

Fig. 3 Cerebral regions significantly activated during memory encoding $(P<0.001)$. (A) Regions activated during encoding in the presence of a difficult distracting task: $(M+D+)-(M-D+)$. (B) Regions activated during encoding in the presence of an easy distracting task: $(M+D-)-(M-D-)$. Areas common to both of the distracting tasks are the superior temporal gyrus, Brodmann area $12 /$ $21 / 22$ (labelled 2) and the left anterior cingulate gyrus, area 32 (1). In the presence of the difficult distracting task, the former activation can be seen to be bilateral. In the presence of the easy distractor, only left-sided activation is seen: at a lower threshold for significance $(P<0.01)$ right-sided activation becomes apparent in this comparison. Present only with the easy distractor are the left prefrontal cortex, Brodmann area $46(4)$ and the retrosplenial area of the posterior cingulate cortex, area 31/23 (3).

A
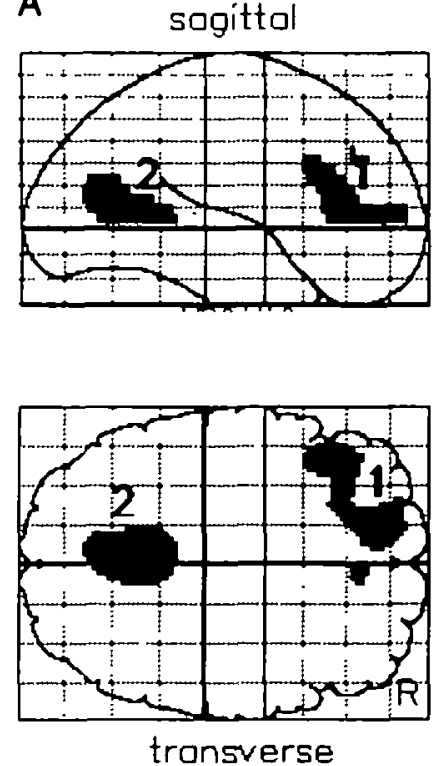

B

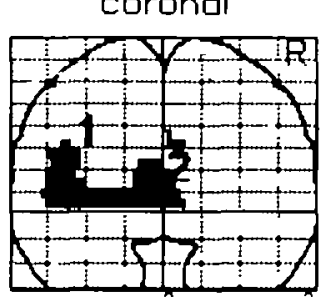

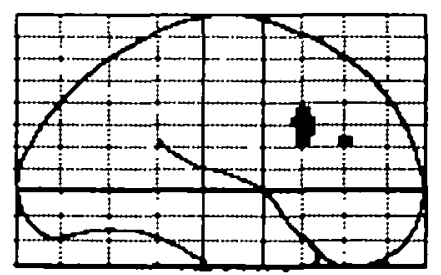

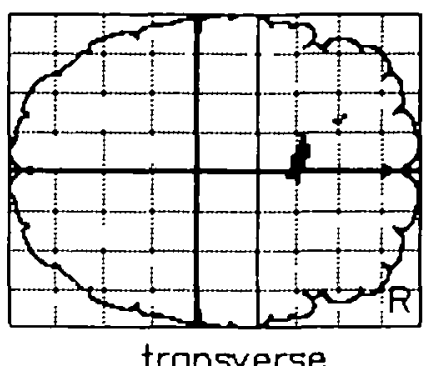

Fig. 4 (A) Regions activated during the encoding of episodic memory in which the activation is attenuated by the performance of a difficult distracting tas: $[(M+D-)-(M-D-)]-[(M+D+)-(M-D+)](P<0.05) .1=$ left prefrontal cortex; $2=$ retrosplenial area of postenor cingulate cortex. (B) Regions activated during the encoding of episodic memory in which the activation is augmented by the performance of a difficult distracting task: $[(M+D+)-(M-D+)]-[(M+D-)-(M-D-)](P<0.05)$. Here the only area seen is the left anterior cingulate cortex. 
Table 1A Experiment 1 : encoding- $r C B F$ increases

\begin{tabular}{|c|c|c|c|c|c|c|}
\hline & $\begin{array}{l}\text { Left superior } \\
\text { temporal }(22)^{*}\end{array}$ & $\begin{array}{l}\text { Right superior } \\
\text { temporal (2l) }\end{array}$ & $\begin{array}{l}\text { Left anterior } \\
\text { cingulate cortex } \\
\text { (32) }\end{array}$ & $\begin{array}{l}\text { Medial frontal } \\
\text { cortex }(9 / 10)\end{array}$ & $\begin{array}{l}\text { Left prefrontal } \\
\text { contex }(46)\end{array}$ & $\begin{array}{l}\text { Posterior cingulate } \\
\text { cortex }(31 / 23)\end{array}$ \\
\hline \multicolumn{7}{|c|}{ Difficult distractor } \\
\hline$x$ & -54 & 48 & -4 & -22 & - & - \\
\hline$y$ & -6 & 4 & 22 & 36 & - & - \\
\hline 2 & 0 & -8 & 28 & 20 & - & - \\
\hline$Z$ value & 5.2 & 4.1 & 5.4 & 4.0 & - & - \\
\hline \multicolumn{7}{|c|}{ Easy distractor } \\
\hline$x$ & -56 & 54 & -2 & - & -32 & -2 \\
\hline$\hat{y}$ & 0 & -8 & 28 & - & 34 & -62 \\
\hline$z$ & -4 & -4 & 28 & 一 & 8 & 12 \\
\hline$Z$ value & 5.0 & 2.5 & 4.0 & - & 4.1 & 4.1 \\
\hline
\end{tabular}

*Corresponding Brodmann areas are given in brackets.

Table 1B Experiment l: encoding-interactions between memory and distractor tasks

\begin{tabular}{|c|c|c|c|}
\hline$x$ & -48 & -6 & - \\
\hline$y$ & 34 & -50 & - \\
\hline$z$ & 8 & 8 & - \\
\hline$x$ & - & - & -12 \\
\hline$y$ & - & - & 0 \\
\hline$z$ & - & - & 32 \\
\hline$Z$ value & - & - & 3.6 \\
\hline
\end{tabular}

Table 2 Experiment 1: encoding-rCBF decreases

\begin{tabular}{|c|c|c|c|c|c|c|c|c|}
\hline & $\begin{array}{l}\text { Right } \\
\text { precuneus }\end{array}$ & $\begin{array}{l}\text { Left } \\
\text { precuneus }\end{array}$ & $\begin{array}{l}\text { Right inferior } \\
\text { parietal }(40)\end{array}$ & $\begin{array}{l}\text { Left inferior } \\
\text { parietal }(40)\end{array}$ & $\begin{array}{l}\text { Right } \\
\text { prefrontal } \\
\text { cortex (44) }\end{array}$ & $\begin{array}{l}\text { Right } \\
\text { premotor (6) }\end{array}$ & $\begin{array}{l}\text { Right } \\
\text { occipital (19) }\end{array}$ & $\begin{array}{l}\text { Left occipital } \\
\text { (19) }\end{array}$ \\
\hline \multicolumn{9}{|c|}{ Difficult distractor } \\
\hline$x$ & 6 & -20 & 44 & -46 & 50 & 26 & - & - \\
\hline$y$ & -58 & -66 & -44 & -28 & 6 & -2 & - & - \\
\hline$z$ & 48 & 44 & 24 & 24 & 16 & 56 & - & - \\
\hline$Z$ value & 3.5 & 4.1 & 3.6 & 4.4 & 4.0 & 4.1 & - & - \\
\hline \multicolumn{9}{|c|}{ Easy distractor } \\
\hline$x$ & 4 & -4 & 38 & -46 & 38 & 10 & 38 & -40 \\
\hline$y$ & -56 & -24 & -28 & -20 & 40 & -14 & -80 & -64 \\
\hline$z$ & 52 & 44 & 32 & 28 & 8 & 48 & 8 & -4 \\
\hline$Z$ value & 4.1 & 5.4 & 6 & 5.9 & 3.8 & 5.3 & 4.2 & 4.1 \\
\hline
\end{tabular}



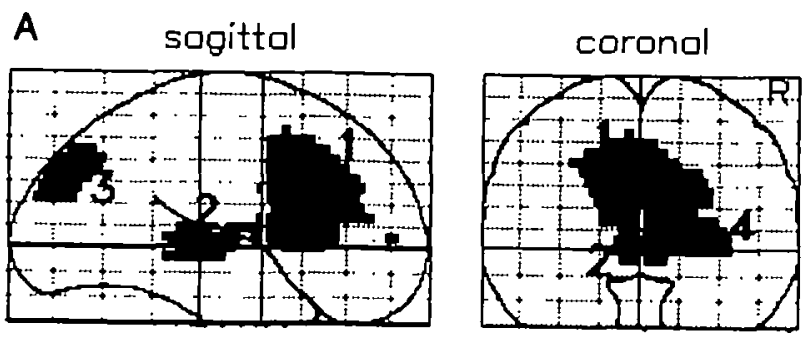

B
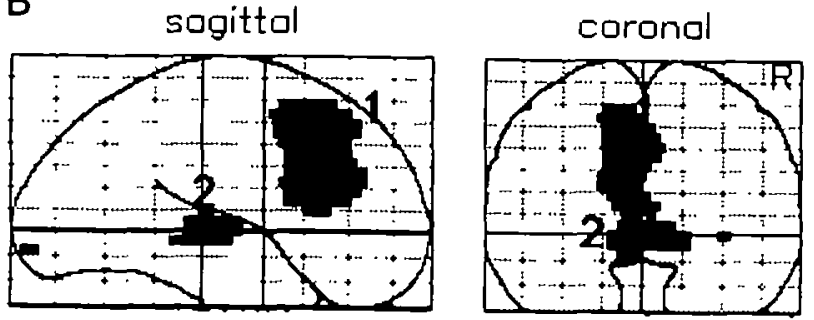
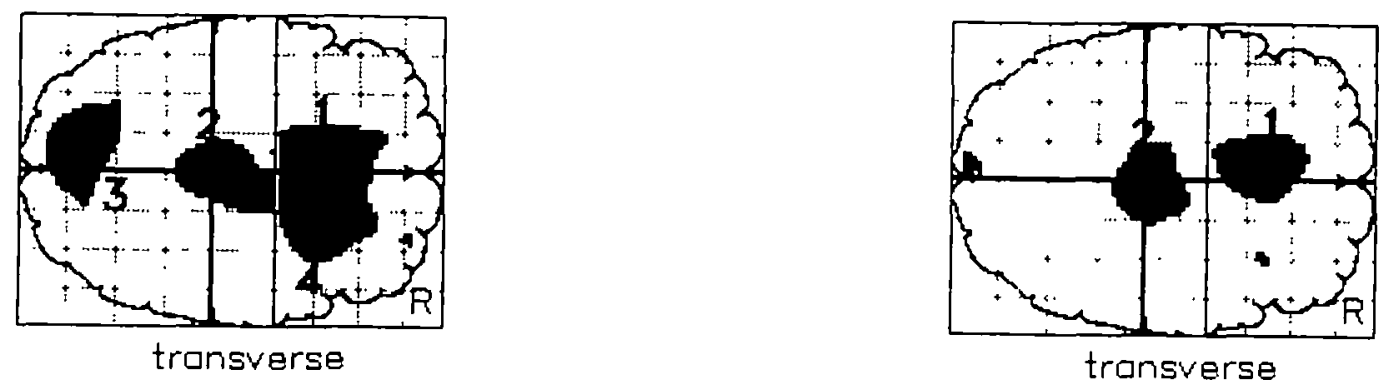

Fig. 5 Cerebral regions significantly activated during memory retrieval ( $P<0.001)$. (A) Episodic memory; (B) semantic memory. Areas common to both episodic and semantic memory are the left anterior cingulate cortex-Brodmann area 32 (1) and the left and right thalami (2). Present to a significantly greater extent in the episodic memory retrieval condition are the precuneus-Brodmann area $7 / 31$ (3) and the right prefrontal region-Brodmann area 10/46/47 (4). There is a small amount of right frontal activation seen in retrieval from semantic memory. This is signıficantly exceeded by that seen in the episodic memory task.

$7: x, y, z=-22,-68,44 ; Z$ value $=2.9$ ). In view of the fact that the resultant difference in activation is very small, we concluded that the performance of the distractor task did not produce a large general activation which would have disguised any activation due to memory encoding.

\section{Experiment 2: retrieval}

\section{$r C B F$ increases}

Episodic memory task compared with the control task. A comparison of scans in which the subjects performed the control (word repetition) task with those involving cued recall of previously presented exemplars showed significant activations in the left anterior cingulate cortex, the right prefrontal cortex, the thalami bilaterally and the precuneus bilaterally. These results are shown as an SPM in Fig. 5A.

Semantic memory task compared with control task. A comparison of control tasks scans with those acquired during performance of semantic memory tasks (self-generated exemplars) revealed significant activations in the left anterior cingulate cortex and thalamus (bilaterally). These data are presented as an SPM in Fig. 5B.

Episodic memory compared to semantic memory. A comparison of scans acquired during episodic with those acquired during semantic memory retrieval tasks revealed significant activations in the precuneus bilaterally and the
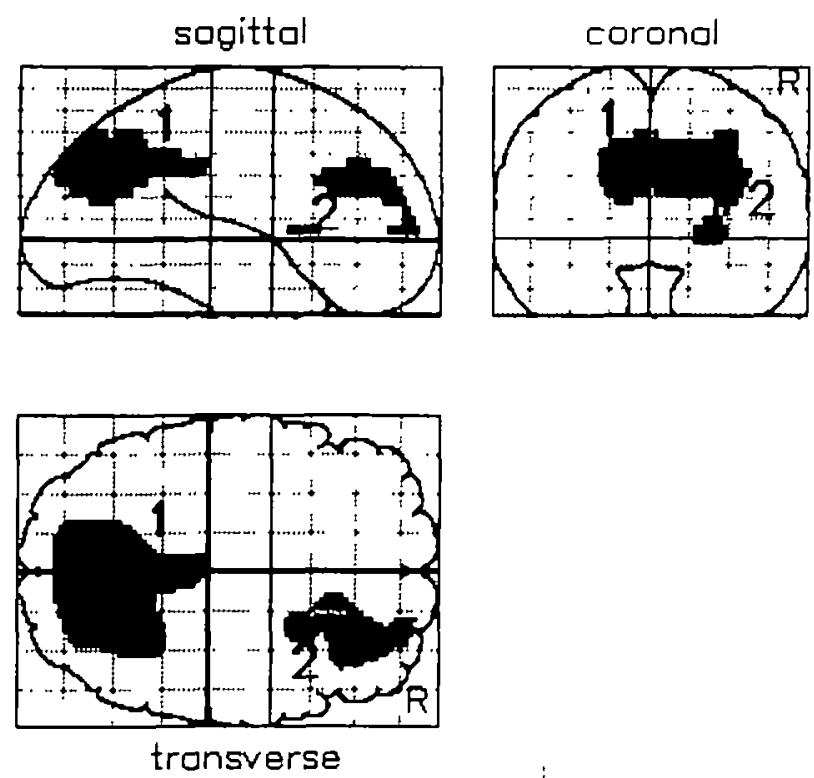

Fig. 6 Regions of significantly greater activation during retrieval from episodic than from semantic memory $(P<0.001) .1=$ precuneus; 2 = right prefrontal cortex.

right prefrontal cortex. These findings are presented as an SPM in Fig. 6.

\section{rCBF decreases}

A comparison showing areas of relative decrease in blood flow was performed for both the episodic and semantic tasks. 
Table 3A Experiment 2: retrieval-rCBF increases

\begin{tabular}{|c|c|c|c|c|c|c|c|}
\hline & $\begin{array}{l}\text { Left anterior } \\
\text { cingulate conex } \\
\text { (32) }\end{array}$ & Right & thalamus & Left thalamus & $\begin{array}{l}\text { Right medial } \\
\text { frontal }(10 / 46)\end{array}$ & Left precuneus (7) & $\begin{array}{l}\text { Right } \\
\text { precuneus (7) }\end{array}$ \\
\hline \multicolumn{8}{|c|}{ Episodıc task } \\
\hline$x$ & -2 & 2 & . & -2 & 18 & -6 & 12 \\
\hline$y$ & 18 & -22 & & -22 & 28 & -68 & -72 \\
\hline$z$ & 36 & 0 & & 8 & 24 & 36 & 28 \\
\hline$Z$ value & 9.4 & 7.5 & & 5.1 & 4.4 & 6.1 & 4.0 \\
\hline \multicolumn{8}{|c|}{ Semantic task } \\
\hline$x$ & -2 & 6 & & -8 & - & - & - \\
\hline$y$ & 20 & -20 & & -24 & - & - & - \\
\hline$z$ & 36 & 0 & & 12 & - & - & - \\
\hline$Z$ value & 6.9 & 6.4 & & 4.1 & - & - & -- \\
\hline
\end{tabular}

Table 3B Experiment 2: retrieval-direct comparison of retrieval tasks

\begin{tabular}{lccc}
\hline \multicolumn{2}{c}{ Right prefrontal cortex $(46 / 10)$} & Left precuneus $(31 / 7)$ & Right precuneus $(31 / 7)$ \\
\hline Episodic versus semantic memory & & \\
$x$ & 30 & -6 & 12 \\
$y$ & 42 & -68 & -72 \\
$Z$ & 24 & 36. & 28 \\
$Z$ value & 4 & 6.1 & 5.6 \\
\hline
\end{tabular}

Table 4 Experiment 2: retrieval-rCBF decreases

Right superior temporal Left superior temporal Right medial temporal Right inferior parietal (40)

(42) (42) (21.37)

\begin{tabular}{|c|c|c|c|c|}
\hline \multicolumn{5}{|c|}{ Episodic task } \\
\hline$x$ & 50 & -50 & 46 & 52 \\
\hline$y$ & -14 & -10 & -60 & -28 \\
\hline$z$ & 8 & 8 & 4 & 28 \\
\hline$Z$ value & 10.3 & 10.0 & 5.1 & 7.1 \\
\hline \multicolumn{5}{|c|}{ Semantic task } \\
\hline$x$ & 48 & -54 & 50 & 52 \\
\hline$y$ & -14 & -14 & -56 & -32 \\
\hline$z$ & 8 & 12 & 8 & 28 \\
\hline$Z$ value & 8.4 & 7.2 & 5.9 & 7.9 \\
\hline
\end{tabular}

Relative deactivations were seen in the superior temporal lobes (Brodmann area 42), the middle temporal lobes (Brodmann areas 21 and 37) and the inferior parietal lobes (Brodmann area $40)$ in both episodic and semantic memory retrieval.

The results for experiment 2 , with appropriate coordinates, are summarized in Table $3 \mathrm{~A}$ ( $\mathrm{rCBF}$ increases for episodic and semantic versus control task), Table 3B (episodic versus semantic comparison) and Table 4 (rCBF decreases).

\section{Discussion}

This study has shown that separate neural structures are involved in the encoding and retrieval components of episodic memory. By using control tasks to subtract out those regions associated with priming and with semantic memory, the study has iśolated the regions activated in episodic memory rather than other processes. Regions isolated during encoding of episodic memory were the left prefrontal and the retrosplenial area of the posterior cingulate cortex. By contrast, we found that regions involved in the retrieval of episodic memory were the right prefrontal cortex and the precuneus. Thus we have shown activation of identical areas to those seen in a previous PET study in which a different paradigm for long-term episodic memory was used and was contrasted with short-term memory (rather than the semantic/priming processes employed in this study) and in which encoding and retrieval occurred within the same scanning period (Grasby et al., 1993a). Our study also showed significant activation of the superior temporal gyri (in the encoding experiment), the thalamus (in the retrieval experiment) and the left anterior cingulate cortex (encoding and retrieval) but these activations were not specific to the episodic memory component. 
The method of data analysis employed in this study, cognitive subtraction, involves the comparison, on a pixel by pixel basis, of two brain states. Control tasks are chosen to be as closely related to the index tasks as possible except in regard to the cognitive component under study. In experiment 1 , the control task (passive listening) will engage systems associated with an auditory input in the same way as the encoding task, but, in contrast, these items will make minimal demands upon a memory system. Thus, the subtraction of the control task from the encoding task isolates the system which subserves this function. Similarly, in experiment 2, the control task is matched with the memory retrieval tasks for both verbal input and output. The subtraction of the control task from either of the memory conditions isolates the systems engaged by retrieval either from episodic or from semantic memory.

\section{The use of the dual task design in PET}

In psychology, dual task methodology has been used to provide evidence for the overlap or separation between the processing systems utilized by different tasks (Shallice et al., 1985). The present application is based upon specific findings that a secondary task will interfere with the encoding of episodic memory but not with priming processes (Baddeley et al., 1984; Jacoby et al, 1993). This interference is mirrored at a neurophysiological level by an abolition of memory-induced activation of the left prefrontal and retrosplenial cortex.

A potential pitfall of the dual task approach would be if the secondary task were to produce a degree of cerebral activation which was sufficient to mask any changes due to the memory task, i.e. if rCBF changes induced by performance of the distractor task were of such magnitude and generality that the influence of memory encoding on rCBF in its presence, "'comparison would be nugatory. We therefore performed a comparison of the M-D+ and M-D-conditions; if the distractor task alone led to a prohibitive degree of activation, we would expect a large difference between these conditions. Since the resultant difference in activation was very small, this indicates that the $M+D+$ versus $M-D+$ comparison was not subject to a serious ceiling effect.

Our finding, of an attenuation of memory-associated prefrontal activation in association with the distractor task, is also of interest in light of recent work showing that such a task preferentially interferes with those aspects of psychological tasks thought to be mediated by prefrontal function (M. Moscovitch, personal communication).

In both experiments, the presentation of category-exemplar paired associates may result in priming as well as episodic memory processes. We used the dual task method to isolate the episodic component. We also sought to minimize the influence of priming (in both experiments 1 and 2) by choosing low frequency category-exemplar pairs and giving subjects explicit memory instructions prior to scanning. Further evidence that the areas that we have shown to be activated during episodic memory processing are not related to priming effects comes from a previous PET study, in which the effect of a priming task in which subjects completed word stems which they had seen previously as parts of complete words (when compared with completion of word stems which had not previously been presented), was to decrease rCBF in the lingual gyrus (Squire et al., 1992). We therefore feel that the two regions which we have isolated during the encoding experiment (left prefrontal and posterior cingulate cortex) and the two isolated during the retrieval experiment (right prefrontal cortex and precuneus) reflect episodic memory processes rather than priming and semantic memory processes.

\section{The retrosplenial cortex and memory}

The subtraction of the regions engaged by memory in the presence of the difficult distractor from those activated in the presence of the easy distractor showed activation of the left prefrontal cortex and the retrosplenial area of the cingulate cortex, suggesting that these regions are critical to encoding. Clinical evidence supports the importance of the retrosplenial cingulate cortex in memory function (Valenstein et al., 1987; Rudge and Warrington, 1991). An important neural pathway connecting the prefrontal cortex with the hippocampus is via this region (Goldman-Rakic et al., 1984; Goldman-Rakic, 1988). The activation in these regions coupled with the finding that a distracting task, which interfered with encoding, attenuated the activation suggests that the retrosplenial cingulate cortex may have a critical role in episodic memory encoding.

\section{Frontal activations: the role in memory}

In patients with prefrontal lesions, many aspects of mnemonic function are preserved (Mayes, 1988; Petrides, 1989). However, an increasing body of evidence indicates that certain aspects of memory function require the integrity of the frontal lobes. They have been implicated in both encoding (Moscovitch, 1982) and retrieval (Incisa della Rochetta and Milner, 1993) processes. Frontal lesions are associated with deficits in temporal ordering of both recent and remote memories (Moscovitch, 1989). Patients with prefrontal lesions tend to have deficits in free recall of unrelated words but there is, generally, little impairment when encoding and retrieval strategies are supplied (Jetter et al., 1986). Patients with frontal excisions also show a high susceptibility to the effects of proactive interference (Schacter, 1987).

Previous PET studies of the long-term episodic component of auditory-verbal memory have shown bilateral prefrontal activations (Grasby et al., 1993a, b). It should be noted that these studies evaluated activations while subjects were engaged in both encoding and retrieval. Subsequently, investigators have used PET to separate these two stages and recent work has indicated a differential role for the left and right frontal lobes in encoding and retrieval, respectively (Kapur et al., 1994; Tulving et al., 1994). These studies, 
however, used very different types of material at the encoding and retrieval stages. We have shown that, when identical material is encoded or retrieved, there is frontal involvement and, in addition, differential engagement of the left and right frontal cortices during encoding and retrieval. Furthermore, the experimental manipulation of encoding by the dual task abolished the left prefrontal activation. The neuropsychological processes subtended by operations on neural signals in the left prefrontal cortex during encoding can only be speculated upon. It seems plausible that distraction prevents the organization of material necessary for efficient encoding. Such an effect may have been reflected at a neurophysiological level (in the significantly attenuated activation) and at a neuropsychological level (in the significantly impaired memory performance). We believe that the finding of right prefrontal activity in retrieval but not in encoding probably does not reflect that it is specifically the right side that is involved in retrieval per se. Instead, it seems more probable that those processes which predominate in memory retrieval, such as internal monitoring/verification (Shallice, 1988) are manifested in right frontal activity. Evidence for this is a finding that perseveration (which seems to indicate a failure of internal verification/monitoring) may predomintae in right-sided frontal lesions (Stuss et al., 1993). These processes differ from those which predominate at encoding, e,g. organization (Incisa della Rochetta, 1986), which may be manifested in left frontal activity. The finding of a relative decrease in right frontal activity during the encoding task supports this view of differential processing leading to differential engagement of the left and right prefrontal cortex during encoding and retrieval.

It has previously been hypothesized that the left frontal cortex is specifically involved in retrieval since subjects with left frontal damage perform normally on category cued word list recall but poorly when cues are removed (even when an organizational strategy has been supplied at word list presentation) (Incisa della Rochetta and Milner, 1993). This finding seems, prima facie, to be at odds with our findings of left frontal activation during encoding and right frontal activation during retrieval. However, our retrieval paradigm differed from the latter experiment in that it involved recall in response to external cues such that a strategic search requiring left frontally mediated internal generation of categories would not be necessary since, in our experiment, subjects were prompted with categories during retrieval and were required only to produce a single exemplar for each category.

\section{The role of the anterior cingulate cortex in attention to action and response selection}

Activation of the left anterior cingulate cortex was common to both encoding and retrieval. The functions of the anterior cingulate cortex are heterogeneous with functional heterogeneity being mirrored in cytoarchitectural and anatomical heterogeneity (Vogt et al., 1987). The anterior cingulate has extensive cortical, subcortical and spinal anatomical connectivity. In particular, reciprocal connections have been described with the prefrontal and parietal cortices (Petrides and Pandya, 1984; Goldman-Rakic, 1988) and with the superior temporal gyrus (Pandya et al., 1981). This widespread connectivity is therefore consistent with a common activation in encoding and retrieval. From a functional perspective, the anterior cingulate cortex appears to have a pre-eminent role in the maintenance of goal-directed behaviours, particularly those that require the suppression of external or internal interfering influences. Cingulate activations have been observed in previous PET studies that involve the internal generation of action, both motor and verbal (Frith et al., 1991), performance of the Stroop test (Pardo et al., 1990; Bench et al., 1993), selective and divided attention during visual discrimination (Corbetta et al., 1991), auditory-verbal graded response memory tasks (Grasby et al., 1993a) and a verbal memory task requiring subjects to hold up to 10 numbers in mind (Petrides et al., 1993).

The anatomical localizations of maximal cingulate activations in our study, during encoding (in the presence of both the easy and the difficult distraction tasks) and retrieval (of both semantic and episodic memory) correspond closely with those previously reported in a study comparing suband supra-span memory conditions with rest $(-8,22,28$ and $-8,18,32$, respectively, in coordinates from the atlas of Talairach and Toumoux, 1988) (Grasby et al., 1993a). This region is also closely related to the focus of activation found in a study comparing internally generated verbal and motor generation tasks with externally cued control conditions (4, 23, 36 and $-3,16,34$, respectively) (Frith et al., 1991). A common feature of tasks which activate the anterior cingulate cortex is attention to the task in question. Direct support for such a role comes from the finding in experiment 1 , in which anterior cingulate activity was seen in the encoding of episodic memory, in the presence of both an easy and a difficult distraction task when compared with the control (passive listening) task. Moreover, performance of the difficult distraction task (requiring increased attention to action) was associated with a significant augmentation of neural activity solely in the left anterior cingulate cortex (Fig. 3B). These data also provide evidence that the distraction-associated attenuation of prefrontal and retrosplenial activation, during encoding was mediated by increased left anterior cingulate activation. In other words, we could speculate that increasing attention to action was associated with selective engagement of the anterior cingulate and that this engagement has a modulatory effect on neural activity in other cortical regions, in this instance, the prefrontal and retrosplenial cortices.

\section{Thalamic activation and memory}

Thalamic activations were observed when comparing both retrieval tasks with a repetition task, but not in the direct comparison of episodic with semantic memory tasks. Furthermore, it was not activated during encoding. This 
suggests that thalamic activation is common to retrieval from both episodic and semantic components of long-term memory. Lesion data support a role for the thalamus in human memory function (Butters and Stuss, 1989). Radiological evidence has suggested that damage to the mammilothalamic tracts is critical to memory impairments (von Crammon et al., 1985). Previous PET studies have described memory-induced thalamic activation, e.g. a ${ }^{18} \mathrm{FDG}$ study showed significant bilateral reductions of thalamic metabolism in patients with amnesia (Fazio et al., 1992). Thalamic activations have been reported during the performance of both sub- and supra-span memory tasks (Grasby et al., 1993a). The differential engagement of the thalamus in retrieval has not, however, to our knowledge been previously demonstrated. The function of the thalamus may therefore involve interconnecting distributed neural representations during memory retrieval, consistent with anatomical evidence of widespread thalamic connectivity with cortical and sub-cortical structures (Blum et al., 1950; Pribram and Barry, 1956; Amaral, 1987; Vogt et al., 1987).

\section{The role of the precuneus}

Activations were seen in the precuneus (Brodmann area 7) during the episodic memory retrieval task but not in the control or the semantic memory tasks. Little is known concerning either the functions or connectivity of the precuneus. Anatomical evidence indicates prefrontal (Petrides and Pandya, 1984; Goldman-Rakic, 1988), temporal, occipital and thalamic connections (Blum et al., 1950; Pribram and Barry, 1956). Engagement of the precuneus has been reported in a a number of PET studies involving memory. In an experiment comparing free word recall from a supra-span list with that from a sub-span list the precuneus was activated in addition to the frontal lobes and the posterior cingulate cortex (Grasby et al., 1993a). In a study in which subjects were required either to produce numbers between 1 and 10 , in random order without repetition, or to listen to a random recital of these numbers and to identify an omission, activity of the precuneus was seen in both conditions (Petrides et al., 1993). Similarly, an experiment involving learning, retrieval and recognition of visual imagery has described precuneus activation (Roland et al., 1990). Although the role of the precuneus is unclear, the present study strongly indicates a specific role in episodic memory retrieval. Grasby et al. (1993a) remarked on the high imageability of memorized items and speculated that the precuneus may be involved in visual mnemonic strategies. Post hoc questioning of the subjects in our study indicated that most had used imagery when recalling the items in the episodic memory task. In this context, it is noteworthy that lesions to this area in rhesus monkeys produced an impairment in somaesthetic learning but not visual learning (Pribram and Barry, 1956). It can be argued that a requirement in a somaesthetic task is the formation of an image from non-visual sensory input. However, such ideas are speculative and further work is needed in order to clarify the function of this area.

\section{Temporal gyrus: increases and decreases}

Activation of the superior temporal gyri was seen in the encoding task in the presence of both the difficult and the easy distractor, suggesting that it reflects semantic/priming processes. At retrieval, there was strong widespread bitemporal (albeit more widespread on the right, extending into the inferior parietal lobe) deactivations in both the episodic and semantic conditions. The evidence for the role of the temporal lobes in language processing is well established both from lesion (Geschwind, 1970) and functional imaging data (Petersen et al., 1988; Wise et al., 1991). Our finding of activation during encoding and deactivation during retrieval may reflect a predominance of phonological, priming and semantic processes on the initial presentation of the paired associates. When the cue words are presented and a memory-demanding response is required, perhaps these processes are less important with this phenomenon being reflected in the relative temporal deactivations. This explanation would be compatible with a previous PET finding in a verbal fluency task in which word repetition was used as a control (Frith et al., 1991); here bitemporal decreases were also seen during word generation, perhaps reflecting that the control/repetition task involves a strong degree of semantic and phonological analysis, whereas in the fluency task the subject is preoccupied with the demands of generating words.

\section{The absence of hippocampal activation}

The current study did not show any hippocampal activation associated with either the encoding or retrieval conditions. This was also the case in a previous PET study in which these two stages were looked at simultaneously (Grasby et al., 1993a). A number of reasons can be put forward to explain the consistent inability of PET to indicate activation in this region. For example, if the hippocampus is continually active during both the experimental and the control tasks, then a cognitive subtraction technique will fail to demonstrate activation. In a recent experiment, Grasby et al. (1993b) looked at correlations of rCBF with memory performance (rather than memory load) and this graded approach examining brain activity in relation to the memory output rather than the demands of the task demonstrated hippocampal activation. Another possibility is that task-associated activation of the hippocampus is of a magnitude outside the sensitivity of PET (see Rolls and Treves, 1990, for relevant theory). The presence of activation of certain hippocampal cell groups may be associated with decreased activity in other, contiguous cell populations such that, given the spatial resolution of the technique, there is no relative change in overall activity, e.g. inactivation of the medial septum in the rat hippocampus leads to reduced spontaneous firing in the $\mathrm{CA} 3$ region, and, in turn, a reduction in feedforward inhibition such that CAl pyramidal cells will not show the expected reduction in activity associated with such a lesion (Mizumori et al., 1989). The complexity in the interactions within the 
hippocampus may explain why it is not accessible to the relatively low spatial resolution of PET.

In conclusion, our experiments indicate that different brain systems are implicated in the encoding and retrieval of auditory-verbal episodic memories. The left prefrontal cortex and the retrosplenial cortex were found to be significantly activated (compared with control tasks) in the encoding of episodic memory, the right prefrontal cortex and the precuneus in its retrieval. In addition, the work suggests that retrieval from episodic and semantic memory engages overlapping, but dissociable, brain systems. The latter finding provides direct neurophysiological evidence for this taxonomic distinction.

\section{References}

Amaral DG. Memory: anatomical organization of candidate brain regions. In: Mountcastle VB, editor. Handbook of physiology, Sect. 1, Vol. 5, Pt 1. Bethesda (MA): American Physiological Society, 1987: 211-94.

Baddeley AD. Human memory: theory and practice. Boston: Allyn and Bacon, 1990.

Baddeley A, Lewis V, Eldridge $M$, Thomson $N$. Attention and retrieval from long-term memory. J Exp Psychol Gen 1984; 113 : $518-40$.

Battig WF, Montague WE. Category norms for verbal items in 56 categories. J Exp Psychol Monograph 1969; 80 No. 3, Pt 2: I-46.

Bench CJ, Frith CD, Grasby PM, Friston KJ, Paulesu E, Frackowiak RSJ, et al. Investigations of the functional anatomy of attention using the Stroop test. Neuropsychologia 1993; 31: 907-22.

Blum JS, Chow KL, Pribram KH. A behavioral analysis of the organization of the parieto-temporo-preoccipital cortex. J Comp Neurol 1950; 93: 53-100.

Butters N, Stuss DT. Diencephalic amnesia. In: Boller F, Grafman J, editors. Handbook of neuropsychology, Vol. 3. Amsterdam: Elsevier, 1989: 107-48.

Cermak LS, Craik FIM, editors. Levels of processing in human memory. Hillsdale (NJ): Lawrence Erlbaum, 1979.

Corbetta M, Miezin FM, Dobmeyer S, Shulman GL, Petersen SE. Selective and divided attention during visual discriminations of shape. color and speed: functional anatomy by positron emission tomography. J Neurosci 1991: 11: 2383-402.

De Renzi E, Liotti M, Nichelli P. Semantic amnesia with preservation of autobiographic memory: a case report. Cortex 1987; 23: 575-97.

Fazio F, Perani D, Gilardi MC. Colombo F, Cappa SF, Vallar G, et al. Metabolic impairment in human amnesia: a PET study of memory networks. J Cereb Blood Flow Metab 1992; 12: 353-8.

Frackowiak RSJ, Friston KJ. Functional neuroanatomy of the human brain: positron emission tomography-a new neuroanatomical technique. [Review]. J Anat 1994; 184: 211-25.

Friston KJ, Passingham RE, Nutt JG, Heather JD, Sawle GV, Frackowiak RSJ. Localisation in PET images: direct fitting of the intercommissural (AC-PC) line. J Cereb Blood Flow Metab 1989; 9: $690-5$.
Friston KJ, Frith CD, Liddle PF, Dolan RJ, Lammertsma AA, Frackowiak RSJ. The relatıonship between global and local changes in PET scans. J Cereb Blood Flow Metab 1990; 10: 458-66.

Friston KJ, Frith CD, Liddle PF, Frackowiak RSJ. Comparing functional (PET) images: the assessment of significant change. J Cereb Blood Flow Metab 1991; 11 : 690-9.

Frith CD, Friston KJ, Lıddle PF, Frackowiak RSJ. Willed action and the prefrontal cortex in man: a study with PET. Proc R Soc Lond Biol 1991; 244: 241-6.

Furlong EJ. A study in memory: a philosophical essay. London: T. Nelson, 1951.

Geschwind $N$. The organızation of language and the brain. Science 1970; 170: 940-4.

Goldman-Rakic PS. Circuitry of primate prefrontal cortex and regulation of behavior by representational memory. In: Mountcastle VB, editor. Handbook of physiology, Sect. 1. Vol 5, Pt 1. Bethesda (MD): American Physiological Society, 1987; 373-417.

Goldman-Rakic PS. Topography of cognition: parallel distributed networks in primate association cortex. [Review]. Annu Rev Neurosci 1988; 11: 137-56.

Goldman-Rakıc PS, Selemon LD, Schwartz ML. Dual pathways connecting the dorsolateral prefrontal cortex with the hippocampal formation and parahıppocampal cortex in the thesus monkey. Neuroscience $1984 ; 12: 719-43$.

Grasby PM, Frith CD, Friston KJ, Bench CJ, Frackowiak RSJ, Dolan RJ. Functional mapping of brain areas implicated in auditoryverbal memory function. Brain 1993a; 116: 1-20.

Grasby PM, Frith CD, Friston KJ, Frackowiak RSJ, Dolan RJ. Activation of the human hippocampal formation during auditoryverbal long-term memory function. Neurosci Lett 1993b; 163: 185-8

Incisa della Rocchetta A. Classification and recall of pictures after unilateral frontal or temporal lobectomy. Cortex 1986; 22: 189-211.

Incisa della Rocchetta A, Milner B. Strategıc search and retrieval inhibition: the role of the frontal lobes. Neuropsychologia 1993; 31: 503-24.

Jacoby LL, Ste-Marie D, Toth JP. Redefining automaticity: unconscious influences, awareness, and control. In: Baddeley A, Weizkrantz L, editors. Attention: selection, awareness and control. Oxford: Clarendon Press, 1993: 261-82.

Jetter W, Poser U, Freeman RB Jr, Markowitsch HJ. A verbal long term memory deficit in frontal lobe damaged patients. Cortex 1986; 22: $229-42$.

Kapur S, Craik FIM, Tulving E, Wilson AA, Houle S, Brown GM. Neuroanatomical correlates of encoding in episodic memory: levels of processing effect [see comments]. Proc Natl Acad Sci USA 1994; 91: 2008-11. Comment in: Proc Natl Acad Sci USA 1994: 91: 1989-91.

Mayes AR. Human organic memory disorders. Cambridge: Cambridge University Press, 1988.

McCarthy RA, Warrington EK. Cognitive neuropsychology: a clinical introduction. San Diego: Academic Press, 1990.

Milner B. Amnesia following operation on the temporal lobes. In: 
Whitty CWM, Zangwill OL, editors. Amnesia. London: Butterworths, 1966: 109-33.

Mizumori SJY, Bames CA, McNaughton BL. Reversible inactivation of the medial septum: selective effects on the spontaneous unit activity of different hippocampal cell types. Brain Res 1989; 500: 99-106.

Moscovitch M. Multiple dissociations of function in amnesia. In: Cermak LS, editor. Human memory and amnesia. Hillsdale (NJ): Lawrence Erlbaum, 1982: 337-70.

Moscovitch M. Confabulation and the frontal systems: strategic versus associative retrieval in neuropsychological theories of memory. In: Roediger HL, Craik FIM, editors. Varieties of memory and consciousness: essays in honour of Endel Tulving. Hillsdale (NJ): Lawrence Erlbaum, 1989: 133-60.

Nickerson RS, editor. Attention and performance VIII. Hillsdale (NJ): Lawrence Erlbaum, 1980.

Pandya DN, Van Hoesen GW, Mesulam M-M. Efferent connections of the cingulate gyrus in the rhesus monkey. Exp Brain Res 1981; 42: $319-30$.

Pardo JV, Pardo PJ, Janer KW, Raichle ME. The anterior cingulate cortex mediates processing selection in the Stroop attentional conflict paradigm. Proc Natl Acad Sci USA 1990; 87: 256-9.

Parkin AJ. Amnesic syndrome: a lesion-specific disorder? Cortex 1984; 20: 479-508.

Petersen SE, Fox PT, Posner MI, Mintun M, Raichle ME. Positron emission tomographic studies of the cortical anatomy of single word processing. Nature 1988; 331: 585-9.

Petrides M. Frontal lobes and memory. In: Boller F, Grafman J, editors. Handbook of neuropsychology, Vol. 3. Amsterdam: Elsevier, 1989: 75-90.

Petrides M, Pandya DN. Projections to the frontal cortex from the posterior parietal region in the rhesus monkey. J Comp Neurol, 1984; 228: 105-16

Petndes M, Alıvisatos B, Meyer E, Evans AC. Functional activation of the human frontal cortex during the performance of verbal working memory tasks. Proc Natl Acad Sci USA 1993; 90: 878-82.

Pribram HB, Barry J. Further behavioral analysis of parieto-temporopreoccipital cortex. J Neurophysiol 1956; 19: 99-106.

Robb RA, Hanson DP. A software system for interactive and quantıtative visualization of multidimensıonal biomedical images. Australas Phys Eng Sci Med 1991; 14: 9-30.

Roland PE, Gulyas B, Seitz RJ, Bohm CJ, Stone-Elander S. Functional anatomy of storage, recall, and recognition of a visual pattern in man. Neuroreport 1990; 1: 53-6.

Rolls ET, Treves A. The relative advantage of sparse versus distributed encoding for associative neuronal networks in the brain. Network 1990; 1: 407-21.

Rudge $P$, Warrington EK. Selective impairment of memory and visual perception in splenial tumours. Brain 1991; 114: 349-60.

Schacter DL. Memory, amnesia, and frontal lobe dysfunction. Psychobiology 1987; 15: 21-36.
Shallice T. From neuropsychology to mental structure. Cambridge: Cambridge Unıversity Press, 1988.

Shallice T, Fletcher P, Frith CD, Grasby PM, Frackowiak RSJ, Dolan RJ. Brain regions associated with acquisition and retrieval of verbal episodic memory. Nature 1994; 368: 633-5.

Shallice T, Mcleod P, Lewis K. Isolating cognitive modules with dual-task paradigm: are speech perception and production separate processes? Q J Exp Psychol 1985; 37A: 507-32.

Squire LR, Ojemann JG, Miezin FM, Petersen SE, Videen TO, Raichle ME. Activation of the hippocampus in normal humans: a functional anatomical study of memory. Proc Natl Acad Sci USA 1992; 89: 1837-41.

Stuss DT, Alexander MP, Palumbo C, Buckle L, Sayer L, Pogue J. Word list learning in patients with frontal lobe lesions [abstract]. Soc Neurosci Abstr 1993; 19: 409.7.

Talairach J, Tournoux P. Co-planar stereotaxic atlas of the human brain. Stuttgart: Thieme, 1988.

Tulving E. Episodic and semantic memory. In: Tulving E, Donaldson W, editors. Organization of memory. New York: Academic Press, 1972: $381-403$.

Tulving E. Elements of episodic memory. Oxford: Clarendon Press, 1983.

Tulving E, Kapur S, Markowitsch HJ, Craik FIM, Habib R, Houle $S$. Neuroanatomical correlates of retrieval in episodic memory: auditory sentence recognition [see comments]. Proc Natl Acad Sci USA 1994; 91: 2012-15. Comment in: Proc Natl Acad Sci USA 1994; 91: 1989-91.

Valenstein E, Bowers D, Verfaellie M, Heilman KM, Day A, Watson RT. Retrosplenial amnesia. Brain 1987; 110: 1631-46.

Vogt BA, Pandya DN, Rosene DL. Cingulate cortex of the rhesus monkey: 1. Cytoarchitecture and thalamic afferents. J Comp Neurol 1987; 262: 256-70.

von Cramon DY, Hebel N, Schuri U. A contribution to the anatomical basis of thalamic amnesia. Brain 1985; 108: 993-1008.

Warrington EK, Weizkrantz L. Amnesia: a disconnection syndrome? Neuropsychologia 1982; 20: 233-48.

Weizkrantz L. Comparative aspects of studies of amnesia. Philos Trans R Soc London Bıol 1982; 298: 97-109.

Wildt AR, Ahtola OT. Analysis of covariance. Quantitative applications in the social sciences, Vol. 12. Beverly Hills: Sage, 1978.

Wise R, Chollet F, Hadar U, Friston K, Hoffner E, Frackowiak RSJ. Distribution of cortical neural networks involved in word comprehension and word retrieval. Brain 1991; 114: 1803-17

Woods RP, Cherry SR, Mazziotta JC. Rapid automated algorithm for aligning and reslicing positron emission tomography images. J Comput Assist Tomogr 1992; 16: 620-33.

Zola-Morgan SM, Squire LR. The pnmate hippocampal formation: evidence for a time-limited role in memory storage. Science 1990; 250: 288-90.

Received June 16, 1994. Revised October 3, 1994.

Accepted November 14, 1994 


\section{Appendix}

Paired associate list

\begin{tabular}{ll} 
Category & Exemplar \\
\hline Animal & Goat \\
Prime minister & Asquith \\
Butterfly & Fritillary \\
Island & Mauritius \\
Aircraft & Wellington \\
Philosopher & Hume \\
Hat & Trilby \\
Bread & Cottage loaf \\
Vegetable & Leek \\
Precious stone & Sapphire \\
Car & Vauxhall \\
Elective office & Councillor \\
Toy & Top \\
Fruit & Lime \\
Money & Cent \\
\hline
\end{tabular}

